\title{
Superconductivity versus phase separation, stripes, and checkerboard ordering: A two-dimensional Monte Carlo study
}

\author{
Daniel Valdez-Balderas* and David Stroud ${ }^{\dagger}$ \\ Department of Physics, The Ohio State University, Columbus, Ohio 43210, USA
}

(Received 17 August 2005; published 2 December 2005)

\begin{abstract}
Using Monte Carlo techniques, we study a simple model which exhibits a competition between superconductivity and other types of order in two dimensions. The model is a site-diluted $X Y$ model, in which the $X Y$ spins are mobile, and also experience a repulsive interaction extending to one, two, or many shells of neighbors. Depending on the strength and range of the repulsion and spin concentration, the spins arrange themselves into a remarkable variety of patterns at low temperatures $T$, including phase separation, checkerboard order, and straight or labyrinthine patterns of stripes, which sometimes show hints of nematic or smectic order. This pattern formation profoundly affects the superfluid density $\gamma$. Phase separation tends to enhance $\gamma$, checkerboard order suppresses it, and stripe formation increases the component of $\gamma$ parallel to the stripes and reduces the perpendicular one. We verify that $\gamma(T=0)$ is proportional to the effective conductance of a random conductance network whose conductances equal the couplings of the $X Y$ system. Possible connections between the model and real materials, such as single high- $T_{c}$ cuprate layers, are briefly discussed.
\end{abstract}

DOI: $10.1103 /$ PhysRevB.72.214501

PACS number(s): 74.20.De, 74.25.Dw

\section{INTRODUCTION}

Systems with competing interactions have been known to show complex patterns of self-organization. ${ }^{1}$ Examples of such systems in two dimensions include the following: type-I superconducting films in their intermediate state ${ }^{2}$ adsorbed monolayers on surfaces; ${ }^{3}$ monomolecular film at air-water interfaces; ${ }^{4}$ ferrimagnetic garnet films $;{ }^{5}$ and doped antiferromagnets, on which there are a number of reports of magnetic and charge ordering. ${ }^{6-14}$ Corresponding to those experimental reports, there have been a number of theoretical models intended to describe them. For example, numerous authors have studied Ising models that include a short-range (e.g., ferromagnetic) interaction plus a longer-ranged (e.g., antiferromagnetic) interaction. ${ }^{15-24}$ The origin of those interactions vary, depending on the particular system under study. In studies of magnetic films, for instance, the origin of the ferromagnetic term is the exchange interaction between spins, while the antiferromagnetic term has its origin in dipoledipole interactions. In the context of hole-doped antiferromagnets, charge stripes are believed ${ }^{25}$ to originate from a short-ranged tendency of the holes to accumulate in regions of suppressed antiferromagnetism, frustrated by a longranged Coulomb repulsion between them. Other workers ${ }^{26}$ have treated stripe phases in the presence of quenched disorder, or using a continuum approach.

Besides charge stripes, other forms of electronic inhomogeneities at low temperatures have been observed in doped antiferromagnets. For example, granular structures consisting of superconducting domains separated by nonsuperconducting regions have been reported ${ }^{27}$ in scanning tunneling microscopy (STM) studies of underdoped $\mathrm{Bi}_{2} \mathrm{Sr}_{2} \mathrm{CaCu}_{2} \mathrm{O}_{8+x}$; spatial variations of the superconducting energy gap and of the local density of states spectrum has been observed in STM experiments ${ }^{28}$ on optimally doped $\mathrm{Bi}_{2} \mathrm{Sr}_{2} \mathrm{CaCu}_{2} \mathrm{O}_{8+x}$; and studies of lightly doped $\mathrm{Ca}_{2-x} \mathrm{Na}_{x} \mathrm{CuO}_{2} \mathrm{Cl}_{2}$ show that electronic states within certain energy ranges show spatial modulations in the form of checkerboards. ${ }^{29}$
This diversity of electronic structures observed in doped antiferromagnets have prompted us to study an $X Y$ model with annealed disorder, where different kind of geometrical orders might occur, and to try to determine the interplay between those orders and superconductivity. Specifically, we have carried out a Monte Carlo study of a site-diluted, twodimensional $X Y$ model on a square lattice, in which, besides the $X Y$ coupling, there is an additional interaction between mobile spins. We have considered two types of such additional interactions: (i) a screened Coulomb repulsive interaction between spins, and (ii) a repulsive interaction between either nearest-neighbor spins or second-nearest-neighbor spins. We assume that this Hamiltonian governs an annealed system, in the sense that the spins are free to move under the influence of the repulsive interaction. They are also free to order in response to the $X Y$ interaction. $X Y$ models have been used to study systems such as granular superconductors, $, 30,31$ high-temperature bulk superconductors, ${ }^{32}$ two-dimensional superfluids, ${ }^{33}$ and the superfluid transition of helium in porous media. ${ }^{34}$ However, to the best of our knowledge, there are no studies of $X Y$ models with annealed disorder such as the one we present here.

Our main purpose in the present work is to investigate (i) what are the low-temperature geometrical orders that occur in an $X Y$ model with annealed disorder and different types of repulsion between spins, and (ii) how this rearrangement of spins affects the helicity modulus $\gamma$ of the system. First, we calculate the $X Y$ transition temperature for various choices of the parameters. This transition appears to be of the Kosterlitz-Thouless variety, though we have not carried out detailed finite-size scaling tests of this hypothesis. We also calculate the behavior of the helicity modulus $\gamma$, which behaves like a tensor for some of the low-temperature phases. Finally, we determine the temperatures at which other transitions occur. These other transitions are induced by the Ising-like spin-spin repulsion mentioned above, and are of two types: (i) an "antiferromagnetic" transition into a check- 
erboardlike structure for nearest-neighbor repulsion, and (ii) a transition into a stripe phase for second-nearest-neighbor repulsion. In both cases, we find that the transition has a strong effect on $\gamma$, which is described in detail below.

Our calculations are carried out using standard Monte Carlo (MC) simulations. In some cases, we calculate critical values of the parameters that determine the type of lowtemperature structures. We also compare our results to previous studies of site-diluted $X Y$ models with quenched disorder. ${ }^{30}$ The behavior in the quenched and annealed cases is notably different, as discussed further below.

The remainder of this paper is organized as follows. In Sec. II, we describe the studied models. Following this, we give a brief description of our computational method in Sec. III. Our results are presented in Sec. IV, and a discussion of them is given in Sec. V.

\section{MODEL}

We consider two distinct model Hamiltonians. The first model, which we denote model I, consists of a twodimensional (2D) square lattice with a fraction $p$ of sites occupied by planar spins, and the remaining $1-p$ vacant. The spins have fixed length but are characterized by an angle $\theta_{i}$, and are assumed to be described by the following classical Hamiltonian:

$$
H=-\sum_{\langle i, j\rangle} J_{i j} \cos \left(\theta_{i}-\theta_{j}\right)+C \sum_{i \neq j} n_{i} n_{j} \frac{\exp \left(-r_{i j} / r_{c}\right)}{r_{i j}} .
$$

Here $J_{i j}=J>0$ if the sites $i$ and $j$ are both occupied, and $J_{i j}=0$ otherwise. The first sum is taken over distinct pairs of nearest neighbors $\langle i, j\rangle$, while the second sum is taken over all distinct pairs $(i, j)$ of lattice sites separated by a distance $r_{i j}<r_{c u t}$, where $r_{\text {cut }}$ is some cutoff radius, usually taken to be a few times the screening length $r_{c}$. $C$ is a non-negative constant that specifies the repulsion strength, and $n_{i}=1$ or 0 is the number of spins at site $i$. The first term thus corresponds to the standard $X Y$ Hamiltonian in 2D, while the second term represents a screened Coulomb repulsion between the spins, which is independent of the angles $\theta_{i}$ and $\theta_{j}$. Thus, each site has two degrees of freedom: the spin variable $\theta_{i}$, and the occupation number $n_{i}$.

Physically, each site which is occupied by a spin can be interpreted as a mobile, positively charged superconducting domain, and each vacant site as a negatively charged nonsuperconducting region. The angles $\theta_{i}$ of the "spins" represent the phases of the superconducting order parameters in the $i$ th domain. When two superconducting domains are close to each other, they couple via Josephson tunneling, which is the origin of the first term in Eq. (1).

The second term in Eq. (1) originates in the Coulomb repulsion between charged superconducting domains. While this term might appear to violate overall charge neutrality, it is, in fact, consistent with that requirement, as we now show. The screened Coulomb term [the second term in Eq. (1)] should really be written as

$$
H_{\text {Coul }}=\sum_{i \neq j} q_{i} q_{j} \frac{\exp \left(-r_{i j} / r_{c}\right)}{r_{i j}},
$$

where the sums run over all pairs of lattice sites, and charge neutrality requires that $\Sigma_{i} q_{i}=0$. We assume that $q_{i}=q_{S}$ or $q_{I}$ on the superconducting or insulating sites, where $p q_{S}+(1$ $-p) q_{I}=0$. Now note that

$$
\begin{aligned}
H_{\text {Coul }}= & \sum_{i \neq j}\left(q_{i}-q_{I}\right)\left(q_{j}-q_{I}\right) \frac{\exp \left(-r_{i j} / r_{c}\right)}{r_{i j}} \\
& +q_{I} \sum_{i \neq j}\left(q_{i}+q_{j}\right) \frac{\exp \left(-r_{i j} / r_{c}\right)}{r_{i j}}-q_{I}^{2} \sum_{i \neq j} \frac{\exp \left(-r_{i j}\right) / r_{c}}{r_{i j}} .
\end{aligned}
$$

But in this last equation, the second term vanishes because $\sum_{i} q_{i}=0$, while the third is just an additive constant. The summand of the first term is nonvanishing only on the $S$ sites. Thus, $H_{\text {Coul }}$ can be rewritten, to within a constant, as

$$
H_{\text {Coul }}=\sum_{i \neq j} n_{i} n_{j}\left(q_{S}-q_{I}\right)^{2} \frac{\exp \left(-r_{i j} / r_{c}\right)}{r_{i j}} .
$$

This form is indeed equivalent to the second term in Eq. (1) with $C=\left(q_{S}-q_{I}\right)^{2}$.

At high temperatures $T$, the system described by Eq. (1) is expected to be phase incoherent, and the spins will point in random directions. As $T$ is reduced, neighboring spins tend to align with each other in order to minimize the system energy. This short-ranged attraction between spins competes with the longer-ranged screened Coulomb repulsion represented by the second term in the Hamiltonian Eq. (1). Thus, this Hamiltonian is a simple model of a system with competing interactions.

We have also studied a model Hamiltonian ("model II") consisting of an $X Y$ term plus a short-range repulsion:

$$
H=-\sum_{\langle i, j\rangle} J_{i j} \cos \left(\theta_{i}-\theta_{j}\right)+A \sum_{\langle i, j\rangle} n_{i} n_{j}+B \sum_{\langle i, j\rangle^{\prime}} n_{i} n_{j} .
$$

Here the first and second sums are carried out over distinct pairs of nearest neighbors $\langle i, j\rangle$, while the third sum runs over all distinct pairs of second nearest neighbors. $A$ and $B$ are non-negative constants specifying the strength of the nearest-neighbor and second-nearest-neighbor repulsion. As will be shown, Hamiltonian (2) at low $T$ produces patterns similar to those of model I.

We obtain the equilibrium thermodynamics of these two models by treating (1) and (2) classically. At a given $T$, the $\theta_{i}$ 's and $n_{i}$ 's arrange themselves so as to minimize the Helmholtz free energy $F$, subject to the constraint that $\Sigma_{i}\left\langle n_{i}\right\rangle / N$ $=p$, where $p$ is assumed to be specified by the experimental conditions. Since both $\theta_{i}$ and $n_{i}$ are free to change, these Hamiltonians describe systems with annealed disorder. By contrast, in a typical system with quenched disorder, the $n_{i}$ 's are assumed fixed, and only the $\theta_{i}$ 's are free to change. In this quenched case, the last two terms in the Hamiltonian would have the same value for any configuration of the $X Y$ spins, and thus play no role in determining the system thermodynamics. 


\section{COMPUTATIONAL METHOD}

\section{A. Monte Carlo approach}

We have studied models I and II for several different values of the parameters $A, B$, and $C$, and for different spin concentrations $p$, using a Monte Carlo approach. We used a square lattice, generally of size $30 \times 30$ sites, with periodic boundary conditions, using the standard MC Metropolis algorithm. ${ }^{35}$ In all of the studied systems we have set $J_{i j}=1$ if both sites $i$ and $j$ are occupied, and $J_{i j}=0$ otherwise. For model I, we considered annealed systems with $C \geqslant 0$, while for model II, we studied two classes of parameters: (i) a nearest-neighbor repulsion only $(A \geqslant 0 ; B=0)$, and (ii) second-nearest-neighbor repulsion only $(A=0 ; B \geqslant 0)$.

We have also reproduced some previous MC studies of 2D systems with quenched disorder, ${ }^{30}$ in order to compare with our present annealed results. For the quenched systems (in which the spins are distributed randomly at fixed locations on the lattice), we averaged over 20 different quenched disorder realizations, each with a spin concentration $p$. For each disorder realization, we started the MC run with the spins arranged in a random configuration of $\left\{\theta_{i}\right\}$ 's and the system at $T=1.2$ (in units of $J / k_{B}$ ). The system was then cooled in steps of $\Delta T=0.05$, down to $T=0.1$, and $\Delta T$ $=0.025$ for $T \leqslant 0.1$. For each $T$, we carried out $5 \times 10^{4}$ sweeps through the entire lattice, taking averages over the last $2 \times 10^{4}$ sweeps, where each sweep consisted of a MC attempt to vary the angle of each spin.

For systems with annealed disorder, we started the system with the spins in randomly chosen sites on the lattice, with randomly chosen angles $\theta_{i}$, at a starting $T=\max [2 C, 1.2]$ (here $C$ is in units of $J / k_{B}$ ) for Hamiltonian (1), and $T=1.2$ for Hamiltonian (2). The system was then cooled down to a $T=0.025$, in steps of $\Delta T$. For model I, we took $\Delta T=0.1 C$ for $T>1.0, \Delta T=0.05$ for $0.1<T<1$, and $\Delta T=0.025$ for $T$ $<0.1$. For model II, we used the same $\Delta T$ 's as in the quenched case. For each $T$, we carried out a series of $\mathrm{MC}$ sweeps over both spin angles and spin positions. During a sweep over the spin angles, MC changes in the spin angles were attempted, while on sweeps over spin positions, the MC step consisted of an attempt to move each of the vacancies to a randomly chosen occupied site. ${ }^{35}$ After each sweep over spin position, ten sweeps over spin angles were carried out. To find the low- $T$ spin configuration of model I, we took a total of $2 \times 10^{6}$ sweeps over spin angles. But to compute thermal averages for model II, we carried out $1 \times 10^{6}$ sweeps over spin angles, discarding the first $5 \times 10^{5}$ in order to allow the system to equilibrate. Following this relaxation, the $\mathrm{MC}$ thermal averages were carried out every tenth sweep over spin angles, immediately before a sweep over spin positions was carried out.

For model I, the second sum was taken only over those distinct pairs of lattice sites which are separated by $r_{i j}$ $<\min \left[3 r_{c}, L / 2\right]$, where $L$ is the linear dimension of the square MC cell. [Thus, the cutoff radius $r_{\text {cut }}$ discussed below Eq. (1) is the lesser of $3 r_{c}$ and $L / 2$.] This choice of $r_{i j}$ $<L / 2$ is necessary to avoid double counting of pairs in when periodic boundary conditions are used, as in the present calculation.
For both model I and model II, we have calculated several equilibrium quantities. To characterize phase coherence, we computed the diagonal elements $\gamma_{\alpha \alpha}(\alpha=x, y)$ of the helicity modulus tensor $\gamma$. These diagonal elements are the spinwave stiffness constants of the $X Y$ spin system, and, in a superconductor, are proportional to elements of the superfluid density tensor. They are defined as appropriate equilibrium averages over the spin configuration. $\gamma_{x x}$, for example, is defined ${ }^{30}$ by

$$
\begin{aligned}
\gamma_{x x}= & \frac{1}{N}\left\langle\sum_{\langle i, j\rangle}\left(x_{i}-x_{j}\right)^{2} J_{i j} \cos \left(\theta_{i}-\theta_{j}\right)\right\rangle \\
& -\frac{1}{N k_{B} T}\left\langle\left(\sum_{\langle i, j\rangle}\left(x_{i}-x_{j}\right) J_{i j} \sin \left(\theta_{i}-\theta_{j}\right)\right)^{2}\right\rangle \\
& +\frac{1}{N k_{B} T}\left\langle\sum_{\langle i, j\rangle}\left(x_{i}-x_{j}\right) J_{i j} \sin \left(\theta_{i}-\theta_{j}\right)\right\rangle^{2},
\end{aligned}
$$

where $x_{i}$ is the $x$ coordinate of the spin on the lattice site $i, N$ is the total number of lattice sites, and \langle\rangle denotes a canonical average. $\gamma_{y y}$ is defined by the analogous expression with $x_{i}$ replaced by $y_{i}$. In our computations, we have taken the lattice constant $a=1$. To characterize the spin patterns, we have also calculated

$$
S_{1}(\vec{q}, T)=\left\langle|n(\vec{q})|^{2}\right\rangle
$$

and

$$
S_{2}(\vec{q}, T)=\left\langle|n(\vec{q})|^{2}\right\rangle-|\langle n(\vec{q})\rangle|^{2},
$$

where

$$
n(\vec{q})=\sum_{j=1}^{N} n_{j} \exp \left(-i \vec{q} \cdot \vec{r}_{j} \cdot\right),
$$

where $\vec{r}_{j}$ is the position of the $j$ th lattice site. For $\vec{q}=\pi \hat{x} / a$ and $\vec{q}=\pi \hat{y} / a, S_{1}(\vec{q}, T)$ and $S_{2}(\vec{q}, T)$ probe stripe formation in the $y$ and $x$ directions, respectively; for $\vec{q}=\pi(\hat{x}+\hat{y}) / a$, they probe the formation of checkerboard patterns, and for $\vec{q}$ $=2 \pi \hat{x} /\left(N_{x} a\right)$ and $\vec{q}=2 \pi \hat{y} /\left(N_{y} a\right)$ (with $N_{x}$ and $N_{y}$ the number of lattice sites in the $x$ and $y$ directions), they are sensitive to phase separation of the system into large domains of occupied and vacant sites.

\section{B. Special approach for low-temperature helicity modulus}

At low $T, \gamma_{x x}(T)$ and $\gamma_{y y}(T)$ can also be calculated by using a mapping between these quantities and the effective conductances $g_{e, x x}$ and $g_{e, y y}$ of a related conductance network. ${ }^{36}$ Specifically, the helicity modulus $\gamma_{\alpha \alpha}(T \rightarrow 0)$ in the $\alpha$ direction satisfies the following relation:

$$
\frac{\gamma_{\alpha \alpha}(T=0, p)}{\gamma_{\alpha \alpha}(T=0, p=1)}=\frac{g_{e, \alpha \alpha}(p)}{g_{e, \alpha \alpha}(p=1)} .
$$

Here $\gamma_{\alpha \alpha}(T=0, p)$ denotes the $\alpha, \alpha$ component of the helicity modulus tensor at $T=0$ for a diluted arrangement of $X Y$ spins of concentration $p$ and the actual $T=0$ configuration, and $\gamma_{\alpha \alpha}(T=0, p=1)$ is the (isotropic) helicity modulus of the corresponding array at $p=1 . g_{e, \alpha \alpha}(p)$ and $g_{e, \alpha \alpha}(p=1)$ are the 
conductances of a certain conductance network associated with the original array of diluted $X Y$ spins, and are constructed as follows. We associate with each spin an electrical node, and with each coupling constant $J_{i j}$ connecting spins at sites $i$ and $j$ we associate a "conductance" $g_{i j}=J_{i j}$. The effective conductance of this network in the $\alpha$ direction $(\alpha=x$ or $y$ ) is denoted $g_{e, \alpha \alpha}$. Equation (7) allows us to calculate the ratios of helicity moduli at two different concentrations $p$ from the corresponding conductances. Our method of calculating the $g_{e}$ 's needed for this mapping is explained below.

\section{RESULTS AND DISCUSSION}

We have carried out extensive simulations for models I and II, considering systems of lattice size $30 \times 30$, and a range of $A, B, C, r_{c}, r_{c u t}$, and $p$, as described below. We first consider the low- $T(T=0.025)$ spin configurations for systems described by model I. Next, we present a more detailed study of model II results over a range of $T$. While the two models differ in how the repulsive interaction is truncated, several features of the low- $T$ spin configurations prove to be model independent.

\section{A. Model I}

We have studied model I [Hamiltonian (1)] for several values of the screening radius $r_{c}$. Low- $T$ configurations were obtained by annealing the system starting from $T$ $=\max [2 C, 1.2]$ to $T=0.025$, as explained in the previous section. Figure 1 shows the $T=0.025$ spin configurations for $r_{c}$ $=\infty$. This corresponds to a unscreened Coulomb repulsion which is, however, truncated at $r=L / 2$. In this and all later figures, the white and black squares in the lattice correspond to occupied and vacant sites. At all $p$, in the absence of a Coulomb repulsion $(C=0)$, the system phase-separates into regions of occupied and vacant sites. This behavior is expected, since this configuration maximizes the number of nearest neighbors for each spin, and hence minimizes the internal energy.

At $C=1$, phase separation no longer occurs; instead, the spins arrange themselves into long, unidirectional stripes, whose width increases with increasing spin concentration $p$. For $C=1$ and $p=0.3$ and 0.7 , we see a kind of smectic pattern in the stripes: the stripes seem to be arranged into layers which have a characteristic thickness, though the spacing between stripes is not perfectly ordered. Such smectic states have been postulated (in the context of a quantum model) for two-dimensional metallic stripe phases in doped Mott insulators. ${ }^{37}$ This spin arrangement is a compromise between the clustering induced by the short-range attractive $X Y$ interaction and the long-range repulsion produced by the Coulomb interaction. As $C$ is increased further, the system undergoes a characteristic series of morphology changes, from long stripes, to shorter stripelike patterns, and eventually to a checkerboard pattern. At some values of $p$ and large $C$, there are suggestions that the occupied or vacant sites have a nematic order - that is, they are arranged in short stripelike patterns with a preferred direction. At certain values of $p$ and $C$, we also see a pattern of diagonal stripes (at $p=0.7, C=4$ in



FIG. 1. Representative snapshots of the spin configurations at low temperatures $(T=0.025)$ for model $\mathrm{I}$ at various points in the $p-C$ phase diagram. We show results for a system with an unscreened Coulomb repulsion truncated at $r=15 a$. $C$ is the strength of the Coulomb repulsion and $p$ is the spin concentration. A white (black) square is an occupied (vacant) site. Each snapshot was obtained by annealing the system from a $T=\max [2 C, 1.2]$ as described in the text.

Fig. 1). The checkerboard patterns are the state of minimum energy when the Coulomb repulsion is much stronger than the $X Y$ attraction.

We have also sampled the low- $T p-C$ phase diagram of model I for finite $r_{c}$ (screened Coulomb repulsion). Figure 2 shows snapshots of the spin configurations for $r_{c}=7$ and $p$ $=0.5,0.6$ and 0.8 . The destruction of the phase-separated case at $C=0$ (shown only in Fig. 1) by the screened Coulomb repulsion proceeds first by formation of long, elongated domains of the minority component (which, for the cases shown in Fig. 2, are vacancies). These domains can be seen in Fig. 2 for $C=0.1$. As $C$ is increased, those elongated domains become unidirectional stripes [as in $(p=0.5, C=1.0)$, $(p=0.5, C=3.0)$, and $(p=0.7, C=1.0)]$, or long but tortuous stripes which coexist with small blobs $[(p=0.8, A=1.0)]$. As in Fig. 1, a further increase in $C$ causes the stripes to break up and leads, for the largest values of $C$, to checkerboard patterns, of which a clear example is $(p=0.5, A=7.0)$ in Fig. 2.

Figures 3 and 4 show spin configurations analogous to those of Figs. 1 and 2. Once again, there is a characteristic sequence of changes with increasing Coulomb repulsion, from phase separation, to an elongated blob phase, to a striped phase, and finally to checkerboardlike phase. At suit- 


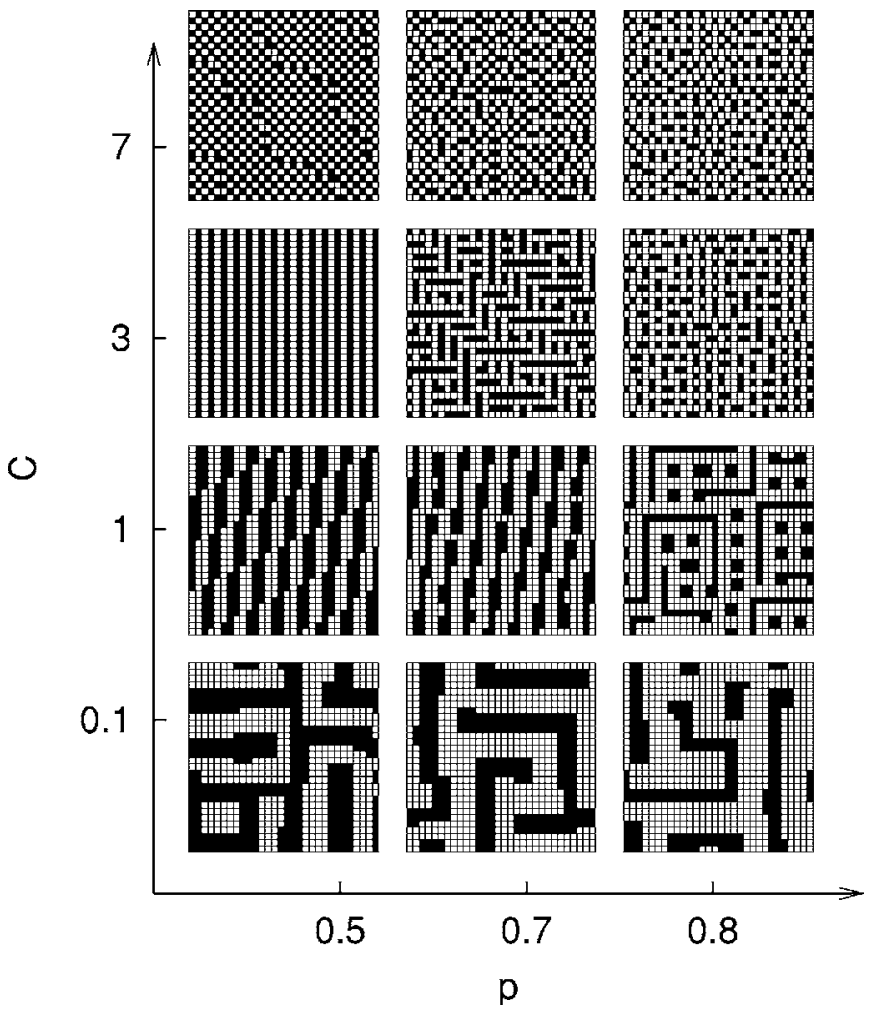

FIG. 2. Same as Fig. 1, but for a screened Coulomb repulsion with $r_{c}=7 a$.

able intermediate values of $C$, certain patterns are strikingly labyrinthine, as seen, for example, in Fig. 2 for $p=0.7$ and

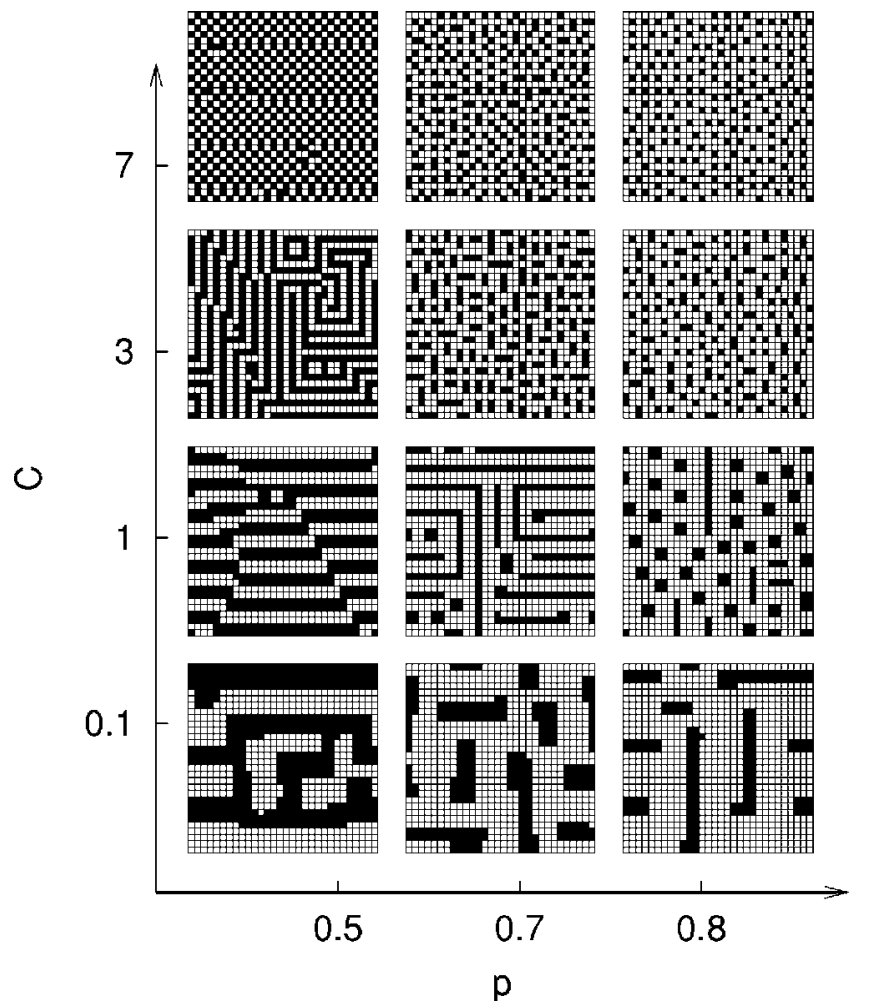

FIG. 3. Same as Fig. 1, but for a screened Coulomb repulsion with $r_{c}=3 a$.



FIG. 4. Same as Fig. 3 but with $r_{c}=a$.

$C=3$, and in Figs. 3 and 4 for $p=0.5$ and $C=3$.

In Table I, we show the diagonal components $\gamma_{x x}$ and $\gamma_{y y}$ of the helicity modulus tensor $\gamma$ for the low- $T$ spin configurations of Fig. 3, as obtained using Eq. (7) introduced above and discussed further below. As may be seen, the checkerboard pattern due to strong Coulomb repulsion dramatically suppresses $\gamma(T)$ at $p=0.5$. Since $\gamma(T)$ is proportional to the superfluid density $n_{s}{ }^{38}$ these results show that a checkerboard pattern, as expected intuitively, strongly suppress the superfluid density. On the other hand, a similarly strong Coulomb repulsion at higher-spin concentrations $(p=0.7$ and $0.8)$ suppresses $\gamma(T)$ only weakly, if at all. This behavior loosely resembles what is seen in doped antiferromagnets, such as the cuprate superconductors, as a function of doping. In this analogy, we can associate the low spin concentration in our model with the underdoped regime of the doped antiferromagnet, where it is believed ${ }^{39}$ that strong Coulomb repulsion leads to an insulating behavior. Likewise, we can associate with the high spin concentration of our model the

TABLE I. Helicity moduli $\left(\gamma_{x x}, \gamma_{y y}\right)$ for a system described by Hamiltonian (1), using $r_{c}=3 a$, at $T=0.025$. $C$ is the strength of the repulsion, and $p$ is the spin concentration. Some snapshots of the system are shown in Fig. 3.

\begin{tabular}{cccc}
\hline \hline$C$ & $p=0.5$ & $p=0.7$ & $p=0.8$ \\
\hline 7 & $(0.00,0.00)$ & $(0.30,0.30)$ & $(0.52,0.51)$ \\
3 & $(0.00,0.07)$ & $(0.37,0.39)$ & $(0.49,0.54)$ \\
1 & $(0.32,0.00)$ & $(0.18,0.00)$ & $(0.42,0.59)$ \\
0.1 & $(0.26,0.00)$ & $(0.29,0.32)$ & $(0.37,0.42)$ \\
\hline \hline
\end{tabular}


overdoped regime of the doped antiferromagnets, where the Coulomb repulsion becomes less important and in which they show more metallic behavior. ${ }^{39}$ (Of course, there is no antiferromagnetism in our model, though there is charge ordering.)

All of the patterns of Figs. 1-4 (stripes, checkerboards, and labyrinths) have been observed, both in experiments and in simulations, in systems with competing long- and shortrange interactions. For example, labyrinthine structures have been observed in experiments on magnetic garnets, ${ }^{40}$ and they have been obtained in simulations of spin-1 Ising Hamiltonians with competing long-range and short-range interactions. ${ }^{24}$ Striped magnetic phases have been observed experimentally in ferrimagnetic garnet films, ${ }^{5}$ and obtained in simulations of spin-1/2 Ising models with a long-ranged dipolar interaction. ${ }^{15}$ Checkerboard patterns in the low- $T$ electronic structure of $\mathrm{Ca}_{2-x} \mathrm{Na}_{x} \mathrm{CuO}_{2} \mathrm{Cl}_{2}$ have been experimentally observed ${ }^{29}$ and also obtained numerically in simulations of a classical spin-1 lattice gas model with shortrange ferromagnetic coupling and long-range antiferromagnetic Coulomb interactions. ${ }^{18}$ There have also been numerical studies ${ }^{16}$ of the stripe melting transition in systems governed by Ising-1/2 models with short ranged ferromagnetic and long ranged antiferromagnetic couplings. The present model differs from all of these in having an $X Y$ rather than an Ising, attractive interaction between the spins. In order to unambiguously distinguish our model from all these others, it would be desirable to carry out simulations on our model I but with an un truncated Coulomb repulsion.

\section{B. Model II}

\section{Numerical results}

In our studies of model II, we have emphasized the $T$ dependence of various quantities, in particular $\gamma(T)$, as well as $S_{1}(\vec{q}, T)$, and $S_{2}(\vec{q}, T)$ for special values of $\vec{q}$. We will also present snapshots of spin configurations at various $T$ 's.

Figure 5(a) shows snapshots of the spin configurations for $p=0.5$ at different $T$ for model II with $A=0, B=0.1$ (weak second-nearest-neighbor repulsion). At $T=1.2$, the spins are already tending to form clusters of various sizes and shapes, a tendency which is clearer at $T=0.5$. At this latter $T$, even though the spins form a connected path in the horizontal $(x)$ direction, $\gamma_{x x}(T)$ [shown in Fig. 5(b)] remains small, indicating no phase coherence. By $T=0.3$, the system has phaseseparated into two large domains, made up of spins and vacancies, respectively. This transition to a phase-separated configuration is signaled by nonzero values of $S_{1}(\vec{q}, T)$ [Fig. $5(\mathrm{c})]$ for $T<0.3$ and by a peak in $S_{2}(\vec{q}, T)$ [Fig. 5(d)] near $T=0.3$, with $\vec{q}=2 \pi \hat{y} / N_{y} a$. The transition to the phasecoherent state, signaled by the finite value of $\gamma(T)$, and that to the phase-separated state, seem to occur at the same $T$.

These results show that the annealed system has strikingly different behavior from a system with quenched disorder. In the latter case, $p=0.5$ is below the critical percolation threshold $p_{c} \simeq 0.59$ for $2 \mathrm{D}$ site-diluted square lattice. ${ }^{41}$ Hence, there is no phase coherence at any $T$ in the quenched case. However, for annealed disorder, the phase separation, which

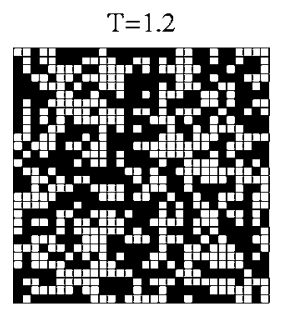

a)


FIG. 5. Some results for model II, with $p=0.5$, and weak second-nearest-neighbor repulsion $B=0.1$. (a) Representative snapshots of spin configurations at different temperatures $T$. White (black) squares are occupied (vacant) sites. (b) Diagonal components $\gamma_{x x}$ and $\gamma_{y y}$ of the helicity modulus tensor, exhibiting a coherence transition. (c), (d) Order parameters sensitive to phase separation, with $q_{x}=2 \pi / N_{x} a$ and $q_{y}=2 \pi / N_{y} a$.

occurs when the Coulomb repulsion is weak enough, leads to an infinite percolating cluster of spins, and hence to phase coherence in one of the two principal directions, even at a spin concentration below $p_{c}$. Although similar phase separation occurs in model I, it does not appear to lead to an infinite cluster of spins for $p<0.5$, and hence, there is no $T=0$ phase coherence in that case.

Figure 6 shows $p=0.5$ for the same model but with a stronger second-neighbor repulsion $(A=0 ; B=1.0)$. Figure 6(a) shows that at $T=1.2$ and especially at $T=0.6$, there is a tendency for stripe formation, though the stripes remain of 




a)


FIG. 6. Results for model II, with $p=0.5$, and strong secondnearest-neighbor repulsion $(B=1.0)$. (a) Representative snapshots of spin configurations at different temperatures $T$. White (black) squares are occupied (vacant) sites. (b) Diagonal components $\gamma_{x x}$ and $\gamma_{y y}$ of the helicity modulus tensor. (c), (d) Order parameters sensitive to stripe formation, with $q_{x}=\pi / a, q_{y}=\pi / a$.

finite length. For $T=0.55$ the system transforms to a mostly unidirectional striped phase. This transition is clearly signaled by a finite value in $S_{1}(\vec{q}, T)$ for $T<0.55$ [Fig. 6(c)], and by a peak in $S_{2}(\vec{q}, T)$ [Fig. $6(\mathrm{~d})$ ] near $T=0.55$, for $\vec{q}=\pi \hat{x} / a$. At $T=0.3$, the system forms an ordered array of stripes in the vertical $(y)$ direction, manifested in the fact that $S_{1}(\vec{q}, T)=1$ at and below this $T$. In contrast to the case of phase separation obtained for weak repulsion in Fig. 5, for which the phase coherence has a sharp onset temperature which coincides with the transition to a phase separated state, the phasecoherent state in the present case has a more gradual onset with decreasing $T$, and occurs at a lower $T$ than the transition

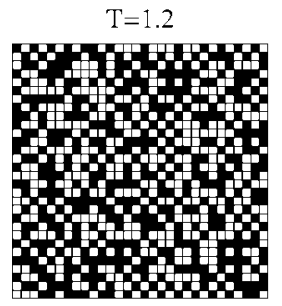

a)

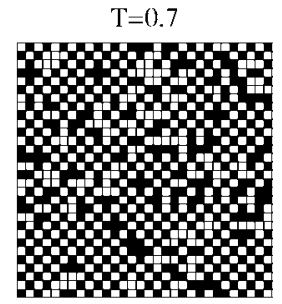

$\mathrm{T}=0.65$

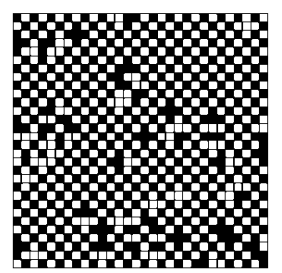

$\mathrm{T}=0.25$


FIG. 7. Results for model II, with $p=0.5$, and a strong nearestneighbor repulsion $(A=1.5)$. (a) Representative snapshots of spin configurations at different $T$. White (black) squares are occupied (vacant) sites. (b) Diagonal components $\gamma_{x x}$ and $\gamma_{y y}$ of helicity modulus tensor. (c), (d) Order parameters sensitive to checkerboard order, with $\vec{q}=\pi(\hat{x}+\hat{y}) / a$.

to a striped phase. Also, the coherence transition is observable in only one of the two principal directions, as expected since phase coherence is geometrically impossible in the direction perpendicular to the stripes.

We turn now to results for model II with nearest-neighbor repulsion only. Figure 7 shows results for $A=1.5, B=0.0$, and $p=0.5$. As in the cases described above, the system goes from a disordered phase at high $T$, to an ordered low- $T$ phase. However, Fig. 7(b) shows that the system is prevented at all $T$ from undergoing a transition with a nonzero $\gamma$. This can be understood on simple geometrical grounds. Figure 7(a) shows that the strong nearest-neighbor repulsion de- 

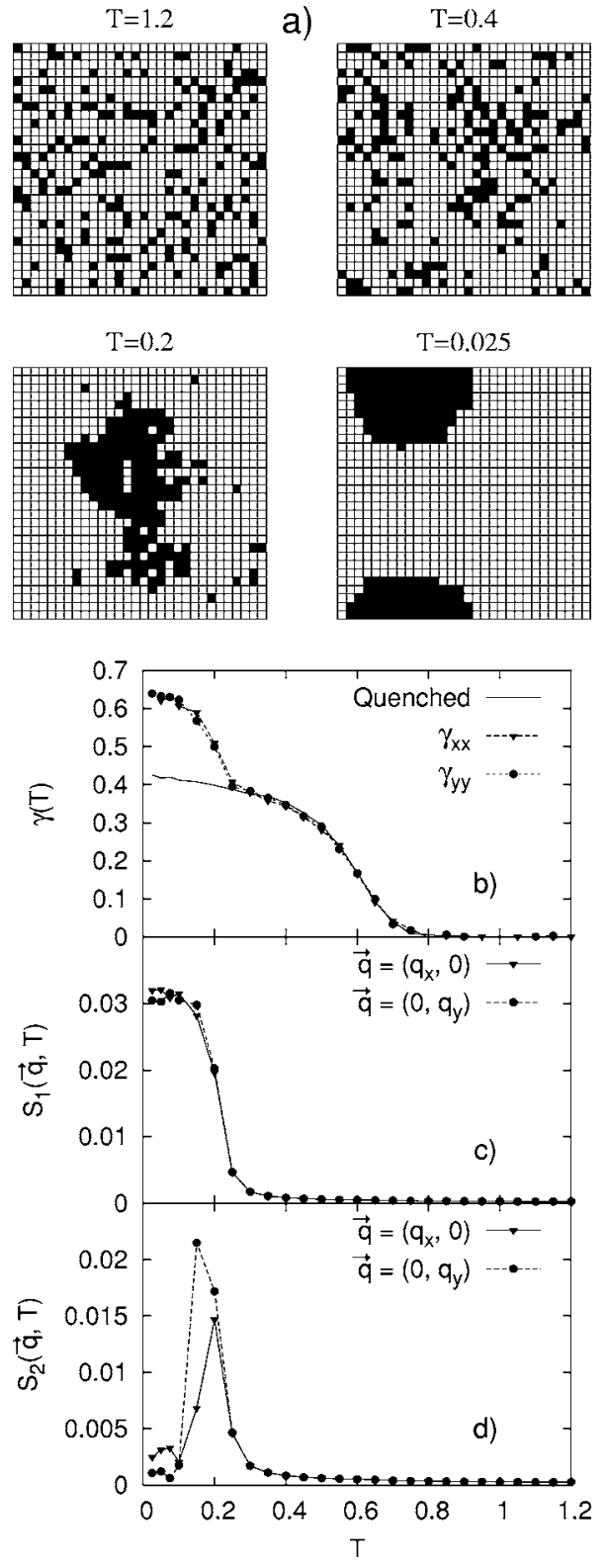

FIG. 8. Results for model II, with $p=0.8$, and weak nearestneighbor repulsion $(A=0.5)$. (a) Spin snapshots at various temperatures $T$. White (black) squares are occupied (vacant) sites. (b) Diagonal components of helicity modulus tensor. (c), (d) Order parameters sensitive to phase separation, with $q_{x}=2 \pi / N_{x} a$ and $q_{y}$ $=2 \pi / N_{y} a$.

creases the number of nearest-neighbor spins at low $T$. Since such nearest-neighbors are essential in for an attractive interactions between the nearest-neighbor $X Y$ spins, this reduction insures that $\gamma(T) \sim 0$, as we observe numerically. The transition to a checkerboardlike spin pattern is indicated by the finite value of $S_{1}(\vec{q}, T)$ for $T<0.65$ and the peak in $S_{2}(\vec{q}, T)$ near $T=0.65$, for $\vec{q}=(\pi / a, \pi / a)$.

We now present results for model II with $p=0.8$. Since $p$ exceeds the site percolation threshold of $p_{c} \simeq 0.59, \gamma(T)$ should become nonzero at sufficiently low $T$. Figure 8 shows results for weak nearest-neighbor repulsion $(A=0.5 ; B=0.0)$, and $p=0.8$. Figure $8(\mathrm{~b})$ shows that $\gamma_{x x}(T) \sim \gamma_{y y}(T)$ for all $T$.
We denote the helicity modulus in this isotropic regime simply as $\gamma(T) . \gamma(T)$ increases smoothly with decreasing $T$ to $T \sim 0.25$, and, in this regime, coincides with $\gamma(T)$ for quenched disorder at same spin concentration [continuous line with no symbols in Fig. 8(b)]. However, the quenched and annealed results differ for $T<0.25$. $\gamma(T)$ for the annealed case changes slope near $T=0.25$ because the spins phase-separate. This phase separation is clearly visible in Fig. 8(a), which shows snapshots of the spin configurations for several values of $T$. It is also signaled by the finite value of $S_{1}(\vec{q}, T)$ for $T \leqslant 0.2$, and a peak in $S_{2}(\vec{q}, T)$ around $T$ $=0.2$ [Figs. 8(c) and 8(d)], for $\vec{q}=2 \pi \hat{x} /\left(N_{x} a\right)$, and $\vec{q}$ $=2 \pi \hat{y} /\left(N_{y} a\right)$.

Figure 9 shows the analogous results for stronger second nearest-neighbor repulsion $(A=0, B=1$, and $p=0.8)$. In this case, the vacancies tend to cluster into stripes, not blobswhich become longer as $T$ is decreased from 1.2 to 0.4 ; below about $T=0.2$, the stripe pattern becomes anisotropic. The helicity moduli $\gamma_{x x}(T)$ and $\gamma_{y y}(T)$ become nonzero around $T=0.7$ but remain isotropic (i.e., nearly equal) down to around $T=0.2$. For $T<0.2$, the anisotropic stripe pattern leads to a dramatic anisotropy in the helicity moduli: $\gamma_{x x}(T)$ falls to 0 , but $\gamma_{y y}(T)$ increases. This behavior has an obvious geometrical explanation in the long stripes parallel to $y$, which inhibit phase coherence in the $x$ direction. At the lowest temperature of $T=0.025$, there are several horizontal stripes spanning the sample, which cause $\gamma_{x x}$ to vanish, while $\gamma_{y y}$ attains a value well above $\gamma(T)$ in the case of quenched disorder. The transition to a striped phase is observed in the onset of a finite value of $S_{1}(\vec{q}, T)$, as well as in the peak in $S_{2}(\vec{q}, T)$, around $T=0.2$, for $\vec{q}=(\pi / a, 0)$. In contrast to the case of weak nearest-neighbor repulsion shown in Fig. 8, where $\gamma(T)$ closely resembles that of the system with quenched disorder for all $T \geqslant 0.25$, the effect of strong second-nearest-neighbor repulsion here leads to a nonmonotonic $\gamma(T): \gamma(T)$ is largest at intermediate $T$, and small at high $T$, where it is destroyed by thermal fluctuations, and at low $T$, where it is frustrated by the formation of stripes. By contrast, at intermediate $T$, short stripes have formed but since they are short and randomly oriented, they are insufficient to prevent a finite $\gamma(T)$.

Finally, we show the results for strong nearest-neighbor repulsion $(A=1.5, B=0, p=0.8)$ in Fig. 10. As in the case of nearest-neighbor repulsion at $p=0.5$ shown in Fig. 7 , where the repulsion prevented phase ordering at all $T, \gamma_{x x}(T)$ and $\gamma_{y y}(T)$ are substantially reduced compared to the corresponding quenched values, and for the same reason: the nearestneighbor repulsion tends to decrease the number of spin nearest neighbors, which in turn decreases the tendency of the system toward phase coherence. The figure also makes apparent that an "antiferromagnetic" (checkerboard) pattern of $n_{i}$ 's is emerging at low $T$, which competes with the $X Y$ transition. But at this $p$, in contrast to $p=0.5$, clumps of checkerboard-ordered regions of zero helicity modulus can coexist with regions of finite helicity modulus, leading to a nonzero global value of $\gamma(T)$.

\section{Analytical results}

At $T=0$, it is possible, by a simple comparison of energies, to calculate analytically the critical values $A_{c}$ and $B_{c}$ at 


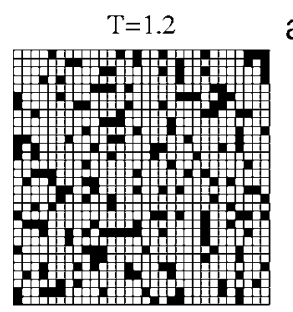

a)
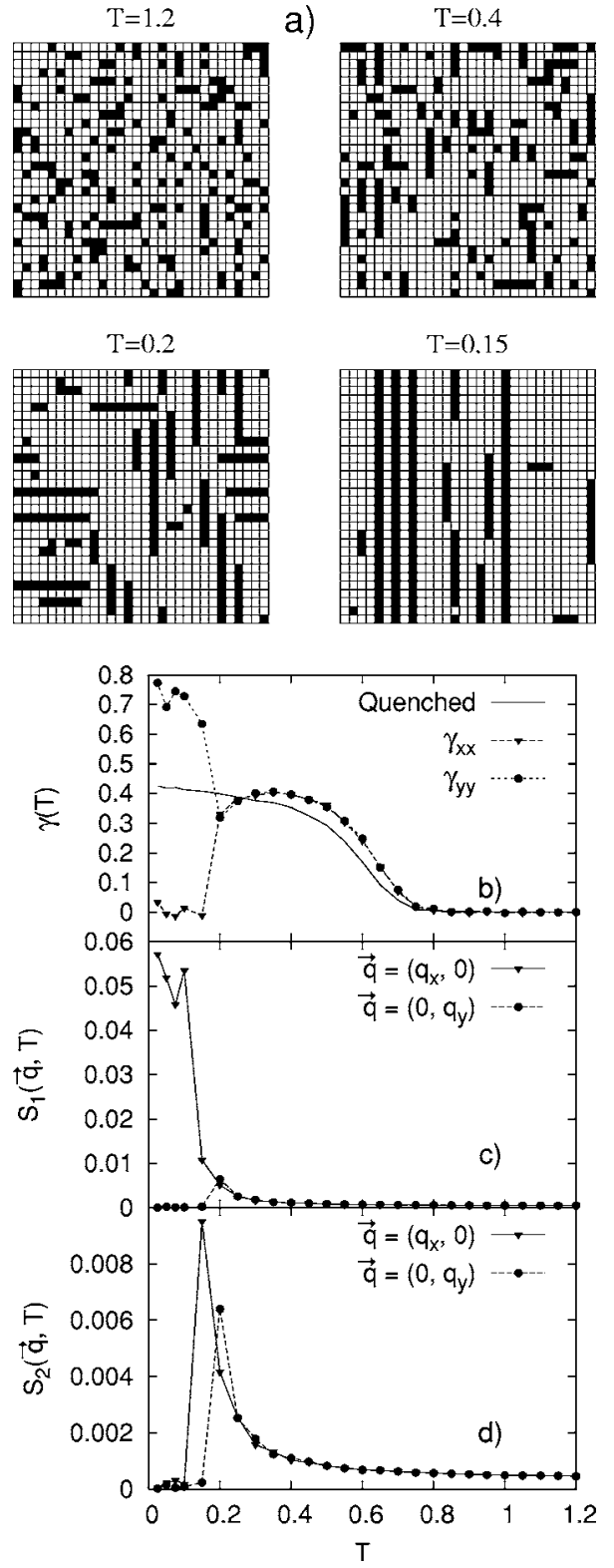

FIG. 9. Results for model II, with $p=0.8$, and strong secondnearest-neighbor repulsion $(B=1.0)$. (a) Spin snapshots at different temperatures $T$. White (black) squares are occupied (vacant) sites. (b) Diagonal components of helicity modulus tensor. (c), (d) Order parameters sensitive to stripe formation, with $q_{x}=\pi / a, q_{y}=\pi / a$.

which the system changes from phase-separated to checkerboard or striped order. In the phase-separated state, all the spins are contained in clusters in which all sites are occupied. Thus, the energy per spin in this state (assuming $B=0$ ) is simply

$$
E^{p s}=-2 J+2 A,
$$

where we have used the fact that each spin has two nearestneighbor spins. For the checkerboard ground state (taking $B=0$ ), the ground-state energy per spin is simply

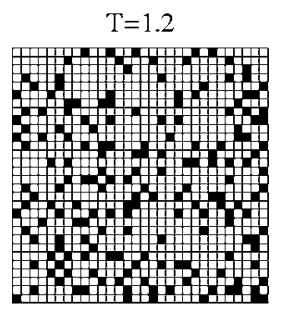

a)

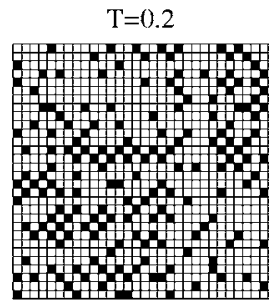

$\mathrm{T}=0.075$

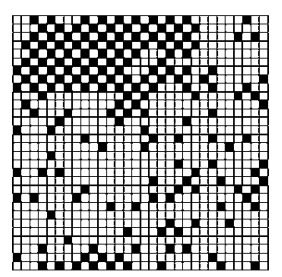

$\mathrm{T}=0.025$
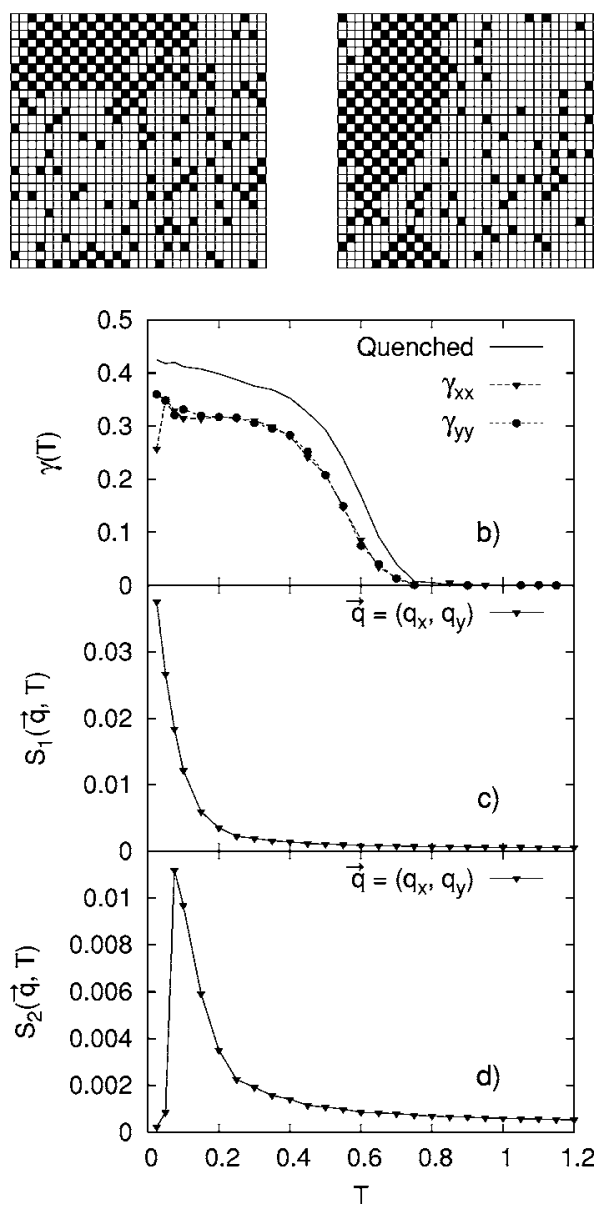

FIG. 10. Model II results, with $p=0.8$ and a strong nearestneighbor repulsion $(A=1.5)$. (a) Spin snapshots at different various temperatures $T$. White (black) squares are occupied (vacant) sites. (b) Diagonal components of the helicity modulus tensor. (c), (d) Order parameters sensitive to checkerboard order, with $\vec{q}=\pi(\hat{x}$ $+\hat{y}) / a$. Note the apparent phase separation at low $T$ into a checkerboard phase and a fully occupied spin phase.

$$
E^{\text {check }}=0 \text {. }
$$

The critical value of $A_{c}$ is just that value of $A$ where the two energies are equal, i.e., $A_{c}=J$. For $A>A_{c}$, the ground state is checkerboard; for $A<A_{c}$, it is phase separated. Our simulations agree with this analytical prediction.

To calculate $B_{c}$, we assume $A=0$ and compare the energies of the two spin arrangements at $T=0$, using a simple bond-counting argument. In the phase-separated state, at $T$ $=0$, the ground state consists of large blobs of spins and vacancies. Disregarding the surface energies, we find that the 
TABLE II. Comparison of the components $\gamma_{x x}(T=0.025, p)$ and $\gamma_{y y}(T=0.025, p)$ for a site-diluted $X Y$ model, as obtained by Monte Carlo simulation of model II, to the effective conductances $g_{e, x x}$ and $g_{e, y y}$ of an associated conductance network, constructed as described in the text. $p$ is the spin concentration while $A(B)$ is the strength of the repulsion between first- (second-) nearest-neighbor spins.

\begin{tabular}{ccccccc}
\hline \hline & & & $\gamma_{x x}(T=0.025, p) /$ & $g_{e, x x}(p) /$ & $\gamma_{y y}(T=0.025, p) /$ & $g_{e, y y}(p) /$ \\
$\gamma_{e, x x}(p=1)$ & $\gamma_{y y}(T=0.025, p=1)$ & $g_{e, y y}(p=1)$ \\
\hline 0.5 & 0.0 & 0.1 & 0.50 & 0.48 & -0.01 & 0.00 \\
0.5 & 0.0 & 1.0 & 0.00 & 0.00 & 0.49 & 0.48 \\
0.5 & 0.5 & 0.0 & 0.50 & 0.48 & 0.01 & 0.00 \\
0.5 & 1.5 & 0.0 & 0.00 & 0.00 & 0.00 & 0.00 \\
0.8 & 0.0 & 0.1 & 0.62 & 0.58 & 0.67 & 0.66 \\
0.8 & 0.0 & 1.0 & 0.03 & 0.00 & 0.77 & 0.76 \\
0.8 & 0.5 & 0.0 & 0.64 & 0.65 & 0.64 & 0.62 \\
0.8 & 1.5 & 0.0 & 0.26 & 0.17 & 0.36 & 0.48 \\
\hline \hline
\end{tabular}

energy per spin is

$$
E^{p s}=-2 J+2 B,
$$

since each spin has two nearest and two second-nearest spin neighbors. In the ground state of the striped phase (at any concentration), we assume that all the spins are contained in clusters consisting of alternating stripes of spins and vacancies. Since each spin in such a cluster has two nearestneighbor spins and no second-nearest-neighbor spins, the ground state energy per spin in this phase is simply

$$
E^{\text {stripes }}=-J .
$$

$B_{c}$ is obtained by setting these two energies equal, which gives $B_{c}=0.5 \mathrm{~J}$. For $B<B_{c}=0.5 \mathrm{~J}$, the ground state is phaseseparated, whereas for $B>B_{c}$, the ground state is striped, independent of $p$.

We have verified this prediction numerically by varying $B$ at fixed $T$ and several values of $p$. In agreement with Eqs. (10) and (11), we find that the system, for any $p$, in the limit of low $T$, always phase separates if $B<B_{c}$ and forms stripes if $B>B_{c}$.

\section{Low-temperature helicity modulus}

For all the low- $T$ configurations shown in Figs. 5-10, we have compared the $\gamma(T)$ 's obtained from Monte Carlo simulations at low $T$ to those obtained from Eq. (7). The configurations are extracted from the snapshots at $T=0.025$, and the effective conductances are calculated by numerically solving the system of linear equations obtained by application of Kirchhoff's equations to each of the nodes in the network. To minimize finite size effects, we used periodic boundary conditions in the direction perpendicular to that for which we calculated the conductances. The diagonalization of the resulting matrix was carried out using Mathematica's built-in function "Solve," which uses the Gaussian elimination method. ${ }^{42}$

In Table II, we show the conductances for these networks, in both the $x$ and $y$ directions, as well as the corresponding values of the helicity moduli at $T=0.025$. Evidently, Eq. (7) is well satisfied for the parameters considered. Where there are discrepancies, we believe that the source of the error is primarily the Monte Carlo simulations, since at very low $T$, most of the attempted Monte Carlo moves are rejected and the phase space sampling may be insufficient to give an accurate equilibrium average. We conclude that our MC simulations are, in general, quite accurately converged, and also that the mapping proposed in Ref. 36 is well obeyed for this rather extensive series of models.

\section{DISCUSSION}

We have studied a diluted $X Y$ model with annealed disorder and an additional spin-spin repulsion. We considered two types of repulsion: (i) a screened Coulomb interaction between spins, with a finite-separation cutoff, and (ii) a shortrange repulsion. In the first case, we have calculated the types of minimum-energy configurations found at low $T$, for a variety of parameter choices. For the second model, we have considered the system at finite and very low $T$.

For the case of Coulomb repulsion (model I), we find that, as the repulsion strength $C$ increased, the system traverses a series of ordered phases in a characteristic sequence: first large blobs (corresponding to a phase-separated state), then horizontal, vertical and diagonal but straight stripes, then tortuous stripes, and finally checkerboardlike patterns. These patterns are strikingly independent of the model details, such how the Coulomb interaction is truncated.

For model II, the low- $T$ spin configuration once again depends on the relative strength of the attractive $X Y$ interaction and the nearest-neighbor or second-nearest-neighbor repulsion. The ground state is always phase separated for weak enough repulsion, but becomes either checkerboard or stripelike for stronger nearest-neighbor or second-nearest-neighbor repulsion. For some concentrations, we see evidence of a phase separation between a checkerboard or striped ordered region, and a region with no vacancies. As $T$ is increased, the spatial ordering of spins, whether phase separated, striped, or checkerboard, eventually disappears in favor of a homogeneous isotropic phase with only limited short-range order. The long but tortuous stripe regime sometimes seen in model 
I at low $T$ appears not to occur with model II, probably because the repulsion is only short range.

Corresponding to these $T$-dependent spin distributions in model II, we have seen characteristic behavior in the helicity modulus $\gamma$. For sufficiently weak repulsion, the system phase separates at low $T$. In this case, for $p>p_{c}$, the two diagonal components of $\gamma$ are approximately equal, and substantially larger than for quenched disorder. For $p<p_{c}$, phase separation leads to a nonzero helicity modulus in only one of the two principal directions. For systems with annealed disorder and strong second-nearest-neighbor repulsion, the formation of short stripes leads, at intermediate temperatures, to an increase of the helicity modulus in both the $x$ and $y$ directions. As $T$ is reduced further, the stripes become longer but remain randomly oriented, leading to a reduction of the helicity modulus in both directions. Finally, as $T \rightarrow 0$, the stripes choose a preferred direction, and the helicity modulus becomes anisotropic, becoming large in the direction parallel to the stripes and very small perpendicular to them. For strong nearest-neighbor repulsion, the helicity modulus is always smaller than in systems with quenched disorder. This decrease is due to the reduction in number of nearest-neighbor spins by the repulsive interaction. In all our calculations, we find good agreement between the low-temperature helicity modulus as obtained from Monte Carlo and that inferred from the conductance of an associated conductance network.

Finally, we comment on the original motivation for this work, which was to shed some light on the interplay between inhomogeneities, stripe, and checkerboard order, and superfluid density in the underdoped cuprate superconductors. Obviously, the present model is far too crude to represent all the subtleties of that system. In particular, it omits quantum effects arising from the noncommutativity of number and phase variables. ${ }^{43}$ But many of the phenomena reported in the cuprates (small superfluid density, frustrated phase separation, and coexistence of superconductivity with other types of order, such as checkerboard or stripe formation) occur in our model. Thus a suitably refined version of the present model might provide insight into the interplay between superconductivity and other collective phenomena in the cuprate superconductors.

\section{ACKNOWLEDGMENTS}

We are grateful for support through the National Science Foundation, Grant No. DMR04-13395. Calculations were carried out using the IA32 Cluster of the Ohio Supercomputer Center.
*Electronic address: balderas@mps.ohio-state.edu

$\dagger$ Electronic address: stroud@mps.ohio-state.edu

${ }^{1}$ Michael Seul and David Andelman, Science 267, 476 (1995).

${ }^{2}$ R. P. Huebener, Magnetic Flux Structures in Superconductors (Springer-Verlag, Berlin, 1979).

${ }^{3}$ Klaus Kern, Horst Niehus, Axel Schatz, Peter Zeppenfeld, Jürgen George, and George Comsa, Phys. Rev. Lett. 67, 855 (1991).

${ }^{4}$ M. Seul and M. J. Sammon, Phys. Rev. Lett. 64, 1903 (1990).

${ }^{5}$ M. Seul and R. Wolfe, Phys. Rev. Lett. 68, 2460 (1992).

${ }^{6}$ S.-W. Cheong, G. Aeppli, T. E. Mason, H. Mook, S. M. Hayden, P. C. Canfield, Z. Fisk, K. N. Clausen, and J. L. Martinez, Phys. Rev. Lett. 67, 1791 (1991).

${ }^{7}$ K. Yamada, C. H. Lee, K. Kurahashi, J. Wada, S. Wakimoto, S. Ueki, H. Kimura, Y. Endoh., S. Hosoya, G. Shirane, R. J. Birgeneau, M. Greven, M. A. Kastner, and Y. J. Kim, Phys. Rev. B 57, 6165 (1998)

${ }^{8}$ J. M. Tranquada, B. J. Sternlieb, J. D. Axe, Y. Nakamura, and S. Uchida, Nature (London) 375, 561 (1995).

${ }^{9}$ T. Niemöller, N. Ichikawa, T. Frello, H. Hünnefeld, N. H. Andersen, S. Uchida, J. R. Schneider, and J. M. Tranquada, Eur. Phys. J. B 12, 509 (1999).

${ }^{10}$ H. A. Mook, Pengcheng Dai, S. M. Hayden, G. Aeppli, T. G. Perring, and F. Dogan, Nature (London) 395, 580 (1998).

${ }^{11}$ M. Arai, T. Nishijima, Y. Endoh, T. Egami, S. Tajima, K. Tomimoto, Y. Shiohara, M. Takahashi, A. Garrett, and S. M. Bennington, Phys. Rev. Lett. 83, 608 (1999).

${ }^{12}$ Pengcheng Dai, H. A. Mook, R. D. Hunt, and F. Dogan, Phys. Rev. B 63, 054525 (2001).

${ }^{13}$ S. M. Hayden, H. A. Mook, Pengcheng Dai, T. G. Perring, and F. Dogan, Nature (London) 429, 531 (2004).

${ }^{14}$ X. F. Sun, Y. Kurita, T. Suzuki, Seiki Komiya, and Yoichi Ando,
Phys. Rev. Lett. 92, 047001 (2004).

${ }^{15}$ I. Booth, A. B. MacIsaac, J. P. Whitehead, and K. De'Bell, Phys. Rev. Lett. 75, 950 (1995).

${ }^{16}$ Antitsa D. Stoycheva and Sherwin J. Singer, Phys. Rev. Lett. 84, 4657 (2000).

${ }^{17}$ A. B. MacIsaac, J. P. Whitehead, M. C. Robinson, and K. De'Bell, Phys. Rev. B 51, 16033 (1995).

${ }^{18}$ U. Löw, V. J. Emery, K. Fabricius, and S. A. Kivelson, Phys. Rev. Lett. 72, 1918 (1994).

${ }^{19}$ Reza Jamei, Steven Kivelson, and Boris Spivak, Phys. Rev. Lett. 94, 056805 (2005).

${ }^{20}$ A. Abanov, V. Kalatsky, V. L. Pokrovsky, and W. M. Saslow, Phys. Rev. B 51, 1023 (1995).

${ }^{21}$ J. Arlett, J. P. Whitehead, A. B. MacIsaac, and K. De'Bell, Phys. Rev. B 54, 3394 (1996).

${ }^{22}$ M. M. Hurley and Sherwin J. Singer, J. Phys. Chem. 96, 1938 (1992).

${ }^{23}$ R. Czech and J. Villain, J. Phys.: Condens. Matter 1, 619 (1989).

${ }^{24}$ J. R. Iglesias, S. Gonçalves, O. A. Nagel, and Miguel Kiwi, Phys. Rev. B 65, 064447 (2002). T. Mertelj, V. V. Kabanov, and D. Mihailovic, Phys. Rev. Lett. 94, 147003 (2005).

${ }^{25}$ See V. J. Emery, S. A. Kivelson, and J. M. Tranquada, Proc. Natl. Acad. Sci. U.S.A. 96, 8814 (1999), and references therein.

${ }^{26}$ C. Reichhardt, C. J. Olson Reichhardt, I. Martin, and A. R. Bishop, Phys. Rev. Lett. 90, 026401 (2003). B. P. Stojkovic, Z. G. Yu, A. L. Chernyshev, A. R. Bishop, A. H. Castro Neto, and Niels Gronbech-Jensen, Phys. Rev. B 62, 4353 (2000). B. P. Stojkovic, Z. G. Yu, A. R. Bishop, A. H. Castro Neto, and Niels Gronbech-Jensen, Phys. Rev. Lett. 82, 4679 (1999).

${ }^{27}$ K. M. Lang, V. Madhavan, J. E. Hoffman, E. W. Hudson, H. Eisaki, S. Uchida, and J. C. Davis, Nature (London) 415, 412 
(2002).

${ }^{28}$ S. H. Pan, J. P. O’Neal, R. L. Badzey, C. Chamon, H. Ding, J. R. Engelbrecht, Z. Wang, H. Eisaki, S. Uchida, A. K. Gupta, K.-W. Ng, E. W. Hudson, K. M. Lang, and J. C. Davis, Nature (London) 413, 282 (2001).

${ }^{29}$ T. Hanaguri, C. Lupien, Y. Kohsaka, D.-H. Lee, M. Azuma, M. Takano, H. Takagi, and J. C. Davis, Nature (London) 430, 1001 (2004).

${ }^{30}$ X. C. Zeng, D. Stroud, and J. S. Chung, Phys. Rev. B 43, 3042 (1991).

${ }^{31}$ W. Y. Shih, C. Ebner, and D. Stroud, Phys. Rev. B 30, 134 (1984).

${ }^{32}$ E. W. Carlson, S. A. Kivelson, V. J. Emery, and E. Manousakis, Phys. Rev. Lett. 83, 612 (1999).

${ }^{33}$ N. Schultka and E. Manousakis, Phys. Rev. B 49, 12071 (1994).

${ }^{34}$ K. Moon and S. M. Girvin, Phys. Rev. Lett. 75, 1328 (1995).

${ }^{35}$ M. E. J. Newman and G. T. Barkema, Monte Carlo Methods in
Statistical Physics (Clarendon Press, Oxford, 1999).

${ }^{36}$ C. Ebner and D. Stroud, Phys. Rev. B 28, 5053 (1983).

${ }^{37}$ V. J. Emery, E. Fradkin, S. A. Kivelson, and T. C. Lubensky, Phys. Rev. Lett. 85, 2160 (2000).

${ }^{38}$ Eric Roddick and David Stroud, Phys. Rev. Lett. 74, 1430 (1995).

${ }^{39}$ E. W. Carlson, V. J. Emery, S. A. Kivelson, and D. Orgad, in The Physics of Superconductors, edited by K. H. Bennemann and J. B. Ketterson (Springer-Verlag, Berlin, 2004), Vol. 2.

${ }^{40} \mathrm{M}$. Portes de Albuquerque and P. Molho, J. Magn. Magn. Mater. 113, 132 (1992).

${ }^{41}$ Muhammad Sahimi, Applications of Percolation Theory (Taylor and Francis, London, 1994).

42 J. E. Gentle, Numerical Linear Algebra for Applications in Statistics (Springer-Verlag, Berlin, 1998).

${ }^{43}$ See, e.g., S. L. Sondhi, S. M. Girvin, J. P. Carini, and D. Shahar, Rev. Mod. Phys. 69, 315 (1997), and references therein. 